\title{
Detention Centers als vernetzte Räume des Einschlusses? Eine gouvernementalitätstheoretische Perspektiverweiterung am Beispiel Lesvos
}

\author{
Tobias Breuckmann \\ Department of Geography, Christian-Albrechts-University, Kiel, 24118, Germany \\ Correspondence: Tobias Breuckmann (breuckmann@geographie.uni-kiel.de)
}

Received: 28 May 2020 - Revised: 4 October 2021 - Accepted: 8 October 2021 - Published: 17 November 2021

\begin{abstract}
Kurzfassung. The article examines the strategic role of detention in the Reception and Identification Center on Lesvos, Greece. Basing on works on detention in carceral geography, I will additionally use the theoretical framework of governmentality. It becomes clear that the detention center on Lesvos serves as a spatial configuration of localization and circulation of asylum seekers framed as belonging to countries with low recognition rate. This is mainly enhanced through confinement, forced or controlled mobilisation as well as the control of flows of assistance and information through containment. In conclusion, certain modes of circulation and mobilization through enclosure can be identified through combination and mutual fertilisation of carceral geography and governmentality.
\end{abstract}

\section{Einleitung}

„You never committed any crime, but they put you to prison anyway“, sagte Amissah ${ }^{1}$ bei meinem Forschungsaufenthalt auf Lesvos im September 2019. Einige Wochen vorher war er nach dreimonatiger Haft aus dem Detention Center des lokalen Hot-Spot-Centers Moria entlassen worden. Ich hatte die Möglichkeit, neben ihm mit mehreren weiteren Personen zu reden, die ähnliche Erfahrungen machten und nach ihrer Ankunft auf Lesvos unmittelbar nach der Registrierung im Hot-Spot-Center für drei Monate inhaftiert wurden. Alle reisten allein, hatten ihre Migrationsrouten in einem westafrikanischen Land begonnen und keine rechtlich verfolgten Straftaten begangen. Aus dieser Tatsache speist sich ihr offensichtlicher Unmut darüber, lediglich für die Einreise mit einer dreimonatigen Haft bestraft zu werden. Wie sich während der Forschung herausstellte, wurden die Inhaftierungen oft auf der Grundlage dessen vollzogen, wie hoch die Behörden die Chancen der Personen auf Asyl bzw. die Flucht- und Verdunkelungsgefahr einschätzten. Stützt man sich bei der Betrachtung dieses Sachverhalts auf die aktuelle Forschung

\footnotetext{
${ }^{1}$ Die interviewten Personen sind im Text durch andere Namen anonymisiert.
}

zu Geflüchtetenlagern, die diese schon seit geraumer Zeit als Sortier- und Regierungsmechanismus von Migration und weniger als Räume des Einschlusses und der Verwahrung sieht (Oesch, 2017:112), drängt sich die Vermutung auf, diese speziellen Hafteinrichtungen nähmen eine bestimmte Funktion innerhalb dieses Mechanismus ein. Auch jüngere geographische Studien, die sich dem Forschungsfeld der carceral geography zuordnen lassen, sehen Detention Centers als Räume, die in unterschiedlichen Kontexten in verschiedene Systeme der Regierung von Migration eingebunden sind (Martin et al., 2019; Conlon und Hiemstra, 2014; Mountz et al., 2012). Dieser empirischen Spur folgend möchte ich unter Hinzunahme des zu Beginn angesprochenen Beispiels eine theoretischkonzeptionelle Rahmung anbieten, die eine geschärfte Analyse der Praktiken des Einschlusses in Detention Centers und deren Einbettung in größere Wirkungszusammenhänge und speziell räumlich benachbarte Techniken erlaubt.

$\mathrm{Zu}$ diesem Zweck stütze ich mich in meiner Forschung auf das Theoriegebäude der Gouvernementalität. In seinen Vorlesungen identifizierte Foucault (2017) einen Übergang von der Disziplinar- zur Sicherheitsgesellschaft und damit einhergehend andere Modi der Regierung, die auf der Sicherstellung und Steuerung der Zirkulation von Menschen und Waren durch die Bearbeitung von Raumproblemen fußen. 
Diese Systeme der Zirkulation erlauben es nach Foucault, bestimmte Formen des Austausches zwischen Raumeinheiten zu forcieren, während unerwünschte Zirkulation durch bestimmte Mechanismen unterbunden bzw. so weit wie möglich verringert werden soll (Foucault, 2017:146). Entsprechend der Konzeption einer Topologie der Macht (vgl. Collier, 2009), laut derer unterschiedliche Techniken und räumliche Ensembles zur Erreichung eines bestimmten Regierungsziels relationiert werden, kann ein für die Disziplinargesellschaft so paradigmatisches Arrangement wie die Haft in liberale Formen der Regierung von Zirkulation eingebunden werden. Die Gouvernementalitätsforschung mit der Migrationsforschung und der carceral geography zu verschränken, kann also zum Verständnis der Einbettung bestimmter Machttechniken in lokale und übergeordnete Systeme der Migrationssteuerung maßgeblich beitragen. Zusätzlich lassen sich vom empirischen Material ausgehend Aussagen darüber treffen, wie bestimmte Personengruppen in gesamtgesellschaftliche Regierungsformen eingebunden werden.

Dafür gehe ich im folgenden Kapitel zunächst auf den Forschungsstand der carceral geography vor allem in Bezug auf das Themenfeld der Mobilität ein. Während allgemein Räume des Einschlusses als vernetzte Praktiken identifiziert wurden, fungieren Geflüchtetenlager als Mechanismen der Einhegung von Mobilität, die wiederum durch verbundene Praktiken der Immobilisierung und Mobilisierung in Gang gesetzt werden sollen. Der Mechanismus der Detention Centers innerhalb des Lagers, um den es im Artikel vornehmlich gehen soll, bildet ein prägnantes Beispiel für einen Raum des Einschlusses. Im Anschluss daran führe ich das Analyseraster der Gouvernementalität detaillierter aus, um dessen Potenziale für die Untersuchung von Haftzentren in Geflüchtetenlagern herauszustellen. Unter gegebenem Analyseraster werden im Empirieteil die Bedingungen und konkreten Techniken in der Haft des Reception and Identification Centers (RIC) auf Lesvos analysiert und ins Verhältnis zu anderen Techniken und übergeordneten Migrationspolitiken gesetzt.

\section{Mobilität als Forschungsfeld der carceral geography}

Schon Mitte der 1970er Jahre bot Foucault eine umfassende Konzeption der Disziplinargesellschaft an, deren offensichtlichste Materialisierung die Ausbreitung der Gefängnissysteme war (Foucault, 2016:935, 1976). Diese sehr allgemeine Beschreibung erfuhr allerdings einige Wendungen und Kritiken (vgl. z. B. Gordon, 1980; Alford, 2000; Hsu, 2003), die ebenfalls in geographischen Studien zu Räumen des Einschlusses Widerhall fanden und in der Geographie im carceral turn mündeten (Moran et al., 2018:666). Im Zuge dessen setzte man sich sowohl mit eher konzeptionellen Fragen und einem analytischen Begriff der carcerality auseinander (Moran et al., 2018:677), als auch mit eher pragmatisch orientierten Fragen wie der Standortwahl bei Gefängnisbauten oder der allgemeinen Eingebundenheit von Räumen des Einschlusses in das nähere und weitere räumliche Umfeld (vgl. Moran et al., 2010).

Ein zentrales Thema der carceral geography bildet das Spannungsfeld zwischen Mobilität und Einschluss: Während bspw. Fishwick und Wearing (2017) darauf verweisen, dass die Räume des Einschlusses der Einhegung einer als schwer zu regierenden Mobilität von Delinquenten dienen (Fishwick und Wearing, 2017:44), stellen Moran et al. (2010:448) die strikte Gegenüberstellung von Mobilität und Inhaftierung infrage und weisen darauf hin, dass erzwungene bzw. disziplinierte Mobilität grundlegend in Straf- und Disziplinarregime eingebunden sein kann. Die Rationalitäten und Technologien der Haft sind also im Spannungsfeld zwischen Fixierung und kontrollierter Mobilisierung angesiedelt (Moran et al., 2010:450), die Haft selbst ist in ein Netzwerk von Institutionen des Strafsystems auf unterschiedlichen sozialräumlichen Ebenen eingebunden (Moran et al., 2018:674). Es sei jedoch weiterhin darauf hingewiesen, dass der Einschluss und die Immobilisierung von Inhaftierten das konstitutive Element der Haft zum Zwecke der Lokalisierung, Isolierung und ggf. Disziplinierung ausmacht, während es immer ein bestimmtes Verhältnis zur Mobilität gibt (Peters und Turner, 2017:2). Aus diesem Grund bezeichnen Peters und Turner (2017) den Zustand des Einschlusses auch als „relative stasis“ (Peters und Turner, 2017:9). Dieses Verhältnis drückt sich nicht lediglich in unterschiedlichen Phasen der Mobilität und Immobilität aus, sondern auch in der eingeschränkten Mobilität im Gegensatz zu anderen am Feld beteiligten Akteur*innen: Dadurch, dass in einem Gefängnis die Vollzugsbeamt*innen eine deutlich höhere Bewegungsfreiheit besitzen, sind Handlungsressourcen extrem ungleich verteilt (Mincke und Lemonne, 2014:532). Es lassen sich also unterschiedliche auf Mobilität bezogene Techniken identifizieren, in die Räume des Einschlusses eingebunden sind: der Einschluss und die Immobilisierung der Inhaftierten und die daraus resultierende strikte Einhegung ihrer Mobilität, die Nutzung der Handlungsressourcen, die sich aus der unterschiedlichen Mobilität der Handelnden ergeben, die erzwungene und einer starken Kontrolle unterworfene Mobilisierung und die damit auf einer übergeordneten Ebene in Beziehung stehende Vernetzung von Orten des Einschlusses.

Im größeren Maßstab lässt sich die Einhegung mobiler Bevölkerungsteile in nationalstaatliche Logiken im Sinne dieser Konzeption anführen. Konkret und besonders prägnant äußert sich dies im Beispiel der Geflüchtetenlager als materialisierte Einhegungsversuche. Neuere Ansätze konzeptualisieren das Geflüchtetenlager zwar, aufbauend auf ersten theoretisch konzeptionellen Ausführungen zu Lagern (vgl. z. B. Agamben, 2002), weiterhin als Ort der angestrebten Exklusion von Asylsuchenden (Martin et al., 2019:2), jedoch auch als umkämpftes sozialräumliches Ensemble der Lenkung, Einhegung und Verlangsamung der Migrationsbewegungen (Oesch, 2017:112). Aufseiten der Regierungshandelnden soll das Lager als Mechanismus der Kontrolle und 
der selektiven Mobilisierung Asylsuchender dienen. Vor allem an den Grenzen soll dort die ungeordnete Migration verlangsamt, gestoppt oder sogar abgewehrt und in Formen der angestrebten Ordnung von Migration überführt werden. Vor Ort agieren sowohl Lagerbetreibende und Außenstehende als auch Geflüchtete auf Basis der materiellen Vorbedingungen des Lagers, die die Handelnden jedoch jeweils für sich strategisch zu nutzen versuchen und so an der Transformation des Raumes teilhaben (Pasquetti, 2015:707). Selbst wenn also das Lager als Kontrollstelle für Migrationsbewegungen dient, sind diese Kontrollen von Brüchen und der Aushandlung zwischen unterschiedlichen Akteur*innen gekennzeichnet (Pasquetti, 2015:707). Andere Autor*innen hingegen heben das erhebliche Macht- und Mobilitätsgefälle zwischen den Handelnden hervor und konzeptualisieren das Lager als „quasi-carceral regime“, da die Mobilität durch die zentrale Unterbringung und Registrierung mit einhergehenden $\mathrm{Zu}$ gangskontrollen und Versorgungsmechanismen in entscheidender Weise eingeschränkt ist (Altin und Minca, 2017:32).

Losgelöst von dieser Diskussion lassen sich sowohl innerhalb des Lagers als auch in der Einbettung des Lagers in größere Zusammenhänge Prozesse der Vernetzung zur Kontrolle der Asylsuchenden identifizieren. So nehmen unterschiedliche - teils miteinander kooperierende, teils konkurrierende - Akteur*innen bestimmte Funktionen innerhalb des Lagers ein. Inhetveen (2014) beschreibt besonders detailliert am Beispiel von Geflüchtetenlagern im südlichen Afrika, wie unterschiedliche NGOs um Arbeits- und Einflussbereiche konkurrieren oder der UNHCR von der Möglichkeit Gebrauch macht, Maßnahmen und Anordnungen von NGOs im Lager zu widerrufen (Inhetveen, 2014:128). Eigene empirische Arbeiten auf Lesvos haben aber auch gezeigt, dass sich unterschiedliche NGOs bei der Unterbringung und der Lebensmittelversorgung Asylsuchender untereinander koordinieren und sowohl die Lagerverwaltung als auch der UNHCR auf Datenbanken einer NGO zurückgreifen, um Aufenthaltsorte von Asylsuchenden abzufragen.

Zudem sind die Akteur*innen und Institutionen wie das Gesamtensemble des Lagers in ihre Umgebung, in Netzwerke der Migration, der Migrationskontrolle und der Versorgung von Migrant*innen sowie in übergeordnete Politiken eingebunden. Das bedeutet aber auch, dass die Betroffenen eben nicht komplett isoliert und marginalisiert werden, sondern die Netzwerke und Beziehungen in ihrem Interesse nutzen können (Inhetveen, 2014:116). Dabei stehen nicht nur formelle Institutionen miteinander in Verbindung, sondern zusätzlich informelle Routen und Orte, wie zum Beispiel an formelle Lager angrenzende informelle Camps, die sich bis zu einem gewissen Grad der Kontrolle entziehen (Martin et al., 2019:4).

Teil dieser eingebetteten Praktiken der Migrationskontrolle sind die Detention Centers. Diese sind laut Conlon und Hiemstra (2014) ein Mechanismus der Migrationskontrolle, der über einen erhöhten Bedarf an nationaler Sicherheit und der Kontrolle ,gefährlicher Körper“ seine Legitimati- on findet (Conlon und Hiemstra, 2014:335). Mountz et al. (2012) schreiben zu Besonderheiten der Detention, dass es sich nur partiell um ein räumliches Ensemble zur Festsetzung der Inhaftierten handle. Vielmehr entstehe auch hier ein Spannungsfeld aus Einschluss und Mobilisierung, teils innerhalb und zwischen verschiedenen Einrichtungen, aber auch zum Zweck der Deportation (Mountz et al., 2012:524). Zusätzlich stellen sie heraus, dass es sich dabei nicht lediglich um die Immobilisierung, sondern um die selektive Kontrolle und Regierung von Migration handle, innerhalb derer bestimmt wird, wer sich frei bewegen darf und wer immobil bleibt bzw. kontrolliert mobilisiert wird (Mountz et al., 2012:526). Auch Mountz und Loyd (2014) gehen in ihrem Artikel zur ,off-shore detention“ in den USA auf vernetzte Praktiken des Einschlusses von Migrant*innen ein. Dort werden auch über größere Distanzen unterschiedliche Orte und Institutionen funktional miteinander in Beziehung gesetzt, sodass sie in ihrer Funktion der Rationalität der Verwaltung von Migrierenden entsprechen (Mountz und Loyd, 2014:390).

Bosworth (2012) plädiert im Kontext von Strafinstitutionen ganz allgemein dafür, diese im Bezugsrahmen von Globalisierung $\mathrm{zu}$ begreifen (Bosworth, 2012:125). Sie weist darauf hin, dass sich die Rolle von Räumen des Einschlusses mit zunehmender Globalisierung und staatlichem Regulationswunsch von Migration dahingehend ändert, dass sie zusätzlich zu Bestrafung und Disziplinierung die Aufgabe der Identifizierung und Kreierung von citizens unter stark modifizierten Bedingungen übernehmen (Bosworth, 2012:126). Zusätzlich falle die aufhebende Wirkung von Strafinstitutionen weg: Die Insass*innen können nicht einfach eine gewisse Zeit absitzen und erhalten damit Zugang zur Staatsbürger*innenschaft, vielmehr bleibt ihr Status als nicht zugehörig von der Haft unberührt (Bosworth, 2012:130).

Die vorangegangenen Ausführungen haben dargelegt, dass Räume des Einschlusses stets ein bestimmtes Verhältnis zur Mobilität einnehmen und dass sie zum Beispiel in Form von Detention Centers eine entscheidende Rolle bei der Einhegung von Migration spielen. Dafür werden die Detention Centers in ein weiteres Netz von Praktiken und Institutionen eingebunden, die sich zu größeren Zusammenhängen der Migrationskontrolle entwickeln und dadurch ihre volle Wirkung entfalten.

Zur klareren Analyse und zur Kontextualisierung dieser Praktiken in größere Zusammenhänge möchte ich das Konzept der Gouvernementalität anbieten, mit dem Foucault Technologien der Regierung analysiert und seine Werkzeuge der Machtanalyse verfeinert hat.

\section{Gouvernementalität und Raum}

Zwischen 1977 und 1979 brachte Foucault in seinen Vorlesungen erstmals den Begriff der Gouvernementalität ins Spiel. In diesem Kontext analysiert er die Vorgänge der Re- 
gierung des Staates seit dem Liberalismus. Dabei beschreibt er die Regierung als das In-Gang-Setzen und die Orchestrierung unterschiedlicher Mechanismen, um bestimmte Formen der Verhaltensführung auf Dauer zu stellen und so Gesamtdynamiken der Bevölkerung beeinflussen zu können (Foucault, 2017:146). Allerdings geht er dazu über, den Begriff der Gouvernementalität und seine Analyse weiter zu fassen bzw. auf mehrere Ebenen zu verlagern. So bildet die Analyse der Rationalitäten des Staates nur eine Analyseebene; die vorausgehend untersuchten Institutionen wie das Gefängnis oder die Produktion des Wahnsinns sind weitere Bausteine einer umfassenden Analyse der Gouvernementalität (Foucault, 2015:261).

Als konstitutiven Modus der Regierung seit dem Liberalismus sieht Foucault das Sicherheitsdispositiv, das den Disziplinarmechanismus als vorherrschende Regierungstechnologie ablöst (Foucault, 2015:73). Während Disziplin „komplementär zur Realität" (Foucault, 2015:76) eingrenzt und direkt auf das Individuum einwirken will, weiten sich Sicherheitsdispositive aus, sie geben die Leitlinien für die Entwicklung einer bestimmten gesellschaftlichen Tendenz vor und wirken nicht ausschweifend disziplinarisch auf das Subjekt ein - sie lassen, ausgehend von der gegebenen Realität, gewähren (Foucault, 2015:73). Es geht also bei der Regierung im Zeichen des Sicherheitsdispositivs darum, Bedingungen zu durchdringen und so in Beziehung bzw. in Kraft zu setzen und die Zirkulation zwischen ihnen sicherzustellen. Dabei soll nicht unterbunden, sondern im Gegenteil allgemein ermöglicht werden, jedoch sollen gleichzeitig Risiken der nicht erwünschten gesellschaftlichen Tendenzen minimiert werden (Foucault, 2015:100). Die Zirkulation beschreibt also - zum Zweck der größtmöglichen Produktivität - einen möglichst ungehinderten Fluss von Personen, Gütern und Kapital, der zwischen unterschiedlichen Einheiten sichergestellt, aber vor allem im Sinne der Regierung gerahmt wird (Forman, 2018:232). Die Zirkulation unterliegt allerdings dem Bestreben und damit teils koordinierter, teils konkurrierender Praktiken verschiedener Akteur*innen, sie möglichst in ihrem Sinne auszugestalten (vgl. Foucault, 2017:146).

Seit Foucault erste Raumprobleme der Regierung identifizierte (Huxley, 2008:1636), haben sich einige Theoretiker*innen an der geographischen Konkretisierung seiner Theorie versucht bzw. sich darum bemüht, Foucaults implizite Konzeptionen von Raum aus seinen Ausführungen herauszuschälen. Philo (1992) leitete aus einzelnen Aufsätzen und der Geschichtsauffassung Foucaults ein Raumkonzept ab, demzufolge sich der Raum aus gleichberechtigten Lagebeziehungen und der Streuung von Beziehungen konstituiert, statt auf übergeordneten und allumfassenden Ordnungssystemen zu basieren. Foucault selbst konstatiert in einem Interview, dass er vornehmlich räumliche Begriffe nutzt, die das Feld der Macht und der Machtbeziehungen abstecken und einordnen (Foucault zit. n. Gordon, 1980:70). Die sich mit sozialräumlichen Beziehungen ko-konstituierenden Machtverhältnisse sind im Kontext des vorliegenden Arti- kels von besonderem Belang, um die konkreten und übergeordneten Systeme der Rahmung von Zirkulation zu analysieren. Füller und Michel (2012) machen diesbezüglich mit ihrer Beschreibung des „Raum[s] als Machttechnik“ deutlich, dass der Raum nicht als Hintergrund von Handlungen konzeptualisiert werden kann, sondern als fundamentaler Teil von sozialen Handlungen und Strategien sowie gesellschaftlichen Strukturierungen anzuerkennen ist (Füller und Michel, 2012:12). Dem folgend erleichtern und erschweren Materialitäten bestimmte aufeinander bezogene Handlungen durch räumliches In-Beziehung-Setzen (Füller und Michel, 2012:12).

So werden Systeme der Zirkulation von Gütern, Kapital und Personen bzw. deren Mobilität über räumlich-materielle Techniken und Strukturen sichergestellt. Über die Veränderung der Komponenten der Zirkulation lassen sich Begebenheiten und Handlungen wahrscheinlicher oder unwahrscheinlicher machen (Marquardt und Schreiber, 2012:41). Dabei stehen Systeme der Zirkulation auf übergeordneter Ebene - wie beispielsweise das europäische Migrationsregime - in einem wechselseitigen Verhältnis mit spezifisch lokalen Techniken der Relationierung von Personen und Materialitäten, die wiederum ihrerseits miteinander in Beziehung gesetzt werden, woraus sich ein umfassenderes System bildet (Salter, 2013:9). Einem topologischen Verständnis von Macht entsprechend werden die Techniken der Regierung von Zirkulationssystemen nicht lediglich von der übergeordneten Ebene ins Lokale überführt. Vielmehr werden schon bestehende Techniken und lokale Gemengelagen so in Beziehung gesetzt und angeeignet, dass sich die Systeme der Zirkulation bestenfalls in die gewünschte Richtung entwickeln. So kommt es, dass die Detention Centers als Räume des Einschlusses flexibel in diese Systeme eingebunden werden können. Dabei sind allerdings - bedingt durch die eigene Materialität der Ensembles und deren Komplexität - bereits potenzielle Brüche in diese Techniken eingeschrieben und bieten die Möglichkeit zu Widerstand und möglicherweise sogar zur Transformation der übergeordneten Systeme (Collier, 2009:90).

Eine dieser Techniken besteht zum Beispiel in der Kontrolle von Abläufen und Bevölkerungsteilen und damit in der Produktion konkreten Wissens über bestimmte Abläufe. Diese Kontrolle wird vor allem über die Lokalisierung ein inhärent räumlicher Begriff - sowie die räumliche Arrangierung zur besseren Lokalisierung gewährleistet (Huxley, 2008:1646). Ein weiteres, sehr anschaulisches Beispiel sind physische Barrieren, die in die Relationierung zwischen Personen einbezogen werden, um auf lokaler Ebene bestimmte Handlungen durchzusetzen (vgl. Breuckmann, 2021). Es macht demnach also bei der geschärften Analyse übergeordneter Systeme der Zirkulation und der Regierung von Mobilität Sinn, nach konkret kleinräumigen Praktiken Ausschau zu halten, die sich, miteinander in Beziehung gesetzt, zu ebendiesen Systemen zusammensetzen. So bieten die vorgestellten Konzeptionen zwei aus meiner Sicht gewinnbringende 
Perspektiven an: Zum einen bietet die Foucault'sche Konzeption der Zirkulation die Möglichkeit, eine systemische Perspektive der Vernetzung und der Einhegung von Mobilität einzunehmen und mit konkreten Techniken in Beziehung zu setzen. Zum anderen eröffnen die Topologien der Macht einen Blick auf konkrete Techniken, die ihre eigene Materialität besitzen und jeweils Beziehungen und Netzwerke herausbilden, aber auch miteinander in Beziehung stehen und so bestimmte Effekte innerhalb eines übergeordneten Systems der Zirkulation erzeugen.

Während im vorliegenden Artikel, aufbauend auf dem Forschungsstand zu Geflüchtetenlagern, angenommen wird, dass das Lager als Mechanismus der selektiven Zirkulation in das Migrationsregime eingebunden ist, konzentriere ich mich empirisch auf den zweiten Punkt - die Topologien der Macht und die wechselseitigen Beziehungen der einzelnen Techniken. Deshalb spezifiziere ich die anfangs aufgeworfene Frage in Bezug auf meine Empirie wie folgt: Wie wird das Detention Center in Moria in den Ordnungsmechanismus Geflüchtetenlager eingebunden und mit anderen lokalen Techniken sowohl innerhalb als auch außerhalb des Lagers in Beziehung gesetzt, um eine selektive Zirkulation von Asylsuchenden durchzusetzen?

Das Ziel der empirischen Analyse ist es also, mehr über die Vorgänge der Zusammensetzung konkreter Techniken zu erfahren, die einen Ordnungsmechanismus auf lokaler Ebene bilden.

\section{Ergebnisse}

Der aufgeworfenen Frage widme ich mich auf Grundlage meiner empirischen Forschung auf der ägäischen Insel Lesvos zu einem der ehemals größten europäischen Geflüchtetenlager, dem Reception and Identification Center Lesvos, besser bekannt als Moria. Nach dem schweren Brand im Lager wurde dieses geschlossen und zum Zeitpunkt des Verfassens dieses Artikels durch ein Übergangslager in der Nähe des alten Camps ersetzt (mare liberum, 2020). Während vier Feldforschungsphasen zwischen September 2018 und April 2020 hatte ich die Möglichkeit, einen tieferen Einblick in die Funktionsweisen des Camps zu bekommen, und zwar hauptsächlich durch teilnehmende Beobachtungen im und um das RIC sowie teilnarrative Interviews mit Geflüchteten, NGO-Mitarbeiter*innen, Anwält*innen und Angehörigen weiterer Institutionen wie der Polizei und des CampManagements. Im September 2019 fanden in diesem Kontext Interviews mit vier ehemals Inhaftierten statt. Die folgenden Ausführungen basieren hauptsächlich auf diesen Interviews, auf Gesprächen mit drei Anwält*innen sowie Dokumenten zum Thema Detention. Auch wenn vor allem aufgrund des erschwerten Zugangs zu Personen - durch eingeschränkte Erreichbarkeit, vor allem aber durch schwer herzustellende Vertrauensverhältnisse - die Anzahl der interviewten ehemals Inhaftierten nicht groß ist, lassen sich aus den Erzäh- lungen und in Verbindung mit Expert*innenwissen und Dokumenten einige Punkte zur adäquaten Analyse der Haft auf Lesvos ausmachen.

\subsection{Tendenzen und Entwicklungslinien der Detention in Griechenland}

Die Inhaftierungsprozeduren von Migrant*innen und Asylsuchenden in Griechenland haben in den letzten Jahren viele Wendungen erfahren und unterliegen vor allem nationalen Politikwechseln, die sich in die Gemengelage europäischer Asylpolitik und damit in Verbindung stehender Migrationsdynamiken einfügen. So hat sich vor allem zu Beginn der 2010er Jahre eine Politik herauskristallisiert, die zum einen den Grenzübertritt nach Griechenland illegalisierte, zum anderen jedoch auf eine Politik der Abschreckung setzte, die zu einer Steigerung der Inhaftierung von Migrant*innen führte. Die Haftbedingungen und -praktiken konsolidierten sich dabei aus dem Spannungsfeld zwischen fingierter Aufnahmebereitschaft bzw. einem Schutzanspruch gegenüber Geflüchteten und einem Sicherheitsdiskurs, der eine gezielte Verschlechterung der Haftbedingungen zur angeblichen Vermeidung von Anreizen für den illegalisierten Grenzübertritt hervorbrachte (Fili, 2018:164f.). Dies ging mit nationalen politischen Bemühungen einher, möglichst alle illegalisiert eingereisten Migrant*innen zu internieren und zu deportieren. Während dies vor allem massenhafte Inhaftierungen auslöste, waren die meisten Deportationen aufgrund fehlender zwischenstaatlicher Abkommen und institutioneller Ressourcen Griechenlands nicht zu gewährleisten. Unter anderem wegen der Finanzkrise wurde das Vorhaben aufgegeben; die damals neue Regierung um die linke Partei Syriza verschrieb sich einem Ende der Inhaftierungs- und Abschiebepraktiken (Fili, 2018:166f.). Die Inhaftierung von Migrant*innen nahm ab 2016, unter derselben Regierung, nach Inkrafttreten des Abkommens zwischen der EU und der Türkei in Griechenland allerdings wieder sukzessive zu, vor allem in Form der Verwaltungshaft (Hänsel, 2019:85f.). Dies lässt sich vor allem als Folge einer neuen Situation nach besagtem Abkommen verstehen: Griechenland und vor allem die Ägäischen Inseln wurden von Transitorten zu Orten der Registrierung und Unterbringung von Geflüchteten, was ein verändertes Verwaltungshandeln notwendig erscheinen ließ (Fili, 2018:180).

Während 2016 noch ein Großteil der Angekommenen für jeweils 25 bis 28 Tage inhaftiert worden war (Hänsel, 2019:89), verschob sich die Praxis der Inhaftierung von gerade angekommenen und registrierten Asylsuchenden egal welcher Herkunft auf allein reisende Männer, die meist aus Staaten mit einer Anerkennungsquote von unter 25 Prozent kamen. Dieses Prozedere wurde mit dem Verfahren des Low Profile Detention Scheme (Saranti, 2019:8) institutionalisiert. Die rechtliche Grundlage dafür bildet das Gesetz „Detention of Applicants", das unterschiedliche Umstände festschreibt, unter denen Migrant*innen in Haft genommen werden können. Grund dafür ist vor allem die vermutete Gefahr, 
die betreffende Person könnte sich dem Asylverfahren entziehen oder es unnötig in die Länge ziehen. Eine Inhaftierung einzig aufgrund eines illegalisierten Grenzübertritts ist hingegen nicht erlaubt (Saranti, 2019:6). Es wird eine Haftdauer von 45 Tagen geplant, bei Revision verlängerbar auf insgesamt 90 Tage (Saranti, 2019). Personen, die einen legitimen Asylgrund vorweisen oder als vulnerabel angesehen werden, sind explizit von der Inhaftierung ausgenommen. Die Feststellung dieser beiden Status ist jedoch lediglich im Zuge der bei der Registrierung vorgesehenen medizinischen Examinierung und des Asylinterviews während der Haft möglich (Saranti, 2019:11).

Obwohl das Prozedere der Inhaftierung bestimmter Zielgruppen also einen Namen bekommen hat und auf ein rechtlich extrem instabiles Fundament gestellt wurde, lässt sich konstatieren, dass bis zur Schließung der Detention infolge des Brandes Unklarheit darüber bestand, wer unter welchen Bedingungen inhaftiert wurde (Fili, 2018:171). Während mir einzelne Inhaftierte berichteten, dass sie das normale Prozedere durchlaufen hätten, wurden ihre Landsmänner ohne stichhaltige Begründung inhaftiert. Der Bericht zur Inhaftierungspraxis auf Lesvos von Saranti (2019) kritisiert explizit, dass den Asylsuchenden keine individuellen Begründungen gegeben wurden (Saranti, 2019:12).

Zusätzlich zu den Verwaltungsinhaftierungen, die einen Großteil der Inhaftierungen darstellten, lassen sich Abschiebeinhaftierungen von Personen, deren Anträge nach einem zweiten Asylverfahren abgelehnt wurden, anführen (Hänsel, 2019:91). Es kam vor, dass Inhaftierte keinen Zugang mehr zu Anwält*innen bekamen, um einen finalen Einspruch gegen die Entscheidung einzulegen, der ihnen jedoch regulär zusteht (Interview mit Anwalt 3). Als dritter Inhaftierungsgrund galt das ,ordnungsverletzende Verhalten“ von Personen, die ohne gerichtliches Verfahren, jedoch durch Anweisung der Polizei auf dem Festland inhaftiert wurden (Hänsel, 2019:92). Im Folgenden wird es vor allem um das Prozedere und die Spezifika der Verwaltungshaft gehen. Zur besseren Einordnung erfolgt zunächst eine kurze Chronologie der Haft von der Inhaftierung bis zur Freilassung, bevor ich auf einzelne Techniken der Regierung der Inhaftierten eingehe und diese zum Lager und konkret zum Asylverfahren in Beziehung setze.

\subsection{Chronologie der Haft in Moria}

In der Regel wurden alle ankommenden Asylsuchenden zur Registrierung in die Reception and Identification Area, kurz New Arrival bzw. RIC Area, gebracht (s. hellblauer Bereich in Abbildung 1). Dort wurden Kurzinterviews zu Fluchtroute und Fluchtgründen durch Mitarbeitende von Frontex und lokalen Polizeibehörden geführt. Abhängig von der Zahl der Ankommenden, der allgemeinen Auslastung der beteiligten Institutionen sowie anderer, weniger vorhersehbarer
Einfussfaktoren ${ }^{2}$ fanden die medizinischen und psychologischen Untersuchungen sowie die Unterbringung entweder unmittelbar nach der Registrierung, nach einigen Tagen oder teilweise auch gar nicht statt. Die schlechte Vorhersehbarkeit und fehlende Regelhaftigkeit der Abläufe war auch der Grund, warum sich die Inhaftierungsabläufe bei den Interviewten teilweise unterschieden. So sind zum Beispiel einzelne Interviewte komplett ohne Registrierung inhaftiert worden, während andere zuvor sogar medizinisch untersucht wurden. Zudem war das Aufnahmeprozedere selbst durch Intransparenz und Inkonsistenz geprägt. Während sich manche Inhaftierte entkleiden mussten und durchsucht wurden, mussten andere dies nicht tun. Von diesem Zeitpunkt an decken sich jedoch die Berichte über die Haft zu großen Teilen. Diese war gekennzeichnet durch unzureichende Versorgung, wenig Platz und kurze Freigänge. Die meisten Inhaftierten führten schon während der Haft ihr erstes Asylinterview durch, sodass sie bereits kurz nach ihrer Entlassung aufgrund der fehlenden Vorbereitung ihre erste Ablehnung erhielten. Im Anschluss an die Haft mussten sie sich eine eigene Unterkunft meist innerhalb des Lagers organisieren, während Registrierte regulär von staatlichen Stellen und NGOs untergebracht wurden.

\subsection{Lokalisierung, Immobilisierung, Parzellierung}

Der offensichtliche und grundlegendste Mechanismus der Inhaftierung lag in der umfassenden Lokalisierung und Immobilisierung der Inhaftierten. Dabei war auch die innere Organisation der Haft durch Parzellierung und teilweise Trennung der Inhaftierten gekennzeichnet. Laut Hänsel (2019) gab es zwei unterschiedliche Sektoren innerhalb der Hafteinrichtung, in denen in den jeweiligen Zellen allein reisende Männer gleicher Nationalität untergebracht wurden (Saranti, 2019:86). Die Hafteinrichtung fasste insgesamt 150 Personen. Mehrere Interviewte berichteten, dass in einer Zelle 14 Personen untergebracht waren, andere Berichte gehen von neun bis zwölf Personen auf $47 \mathrm{~m}^{2}$ aus (Saranti, 2019:15). Insgesamt leistete der schiere Einschluss den Großteil der Bewegungskontrolle. Zudem wurden die Inhaftierten dreimal täglich gezählt, während sie sonst größtenteils sich selbst überlassen wurden. Ato erzählte im Interview, dass die Zellen videoüberwacht worden seien, er jedoch keine diesbezüglichen Auswirkungen im Sinne disziplinarischer Techniken wahrgenommen habe. Zusätzlich bestand die Möglichkeit, Inhaftierte in Isolationshaft unterzubringen. Anfang Januar 2020 wurden Berichte öffentlich, nach denen sich ein Inhaftierter in seiner Zelle suizidierte, nachdem er mehrere Wochen in Isolationshaft gehalten worden sei, obwohl er

\footnotetext{
${ }^{2}$ Beispielsweise unterbrach Kelpno, die mit der medizinischen und psychologischen Untersuchung betraute staatliche Stelle, infolge eines Konflikts mit Asylsuchenden ihre Arbeit für mehrere Wochen.
} 


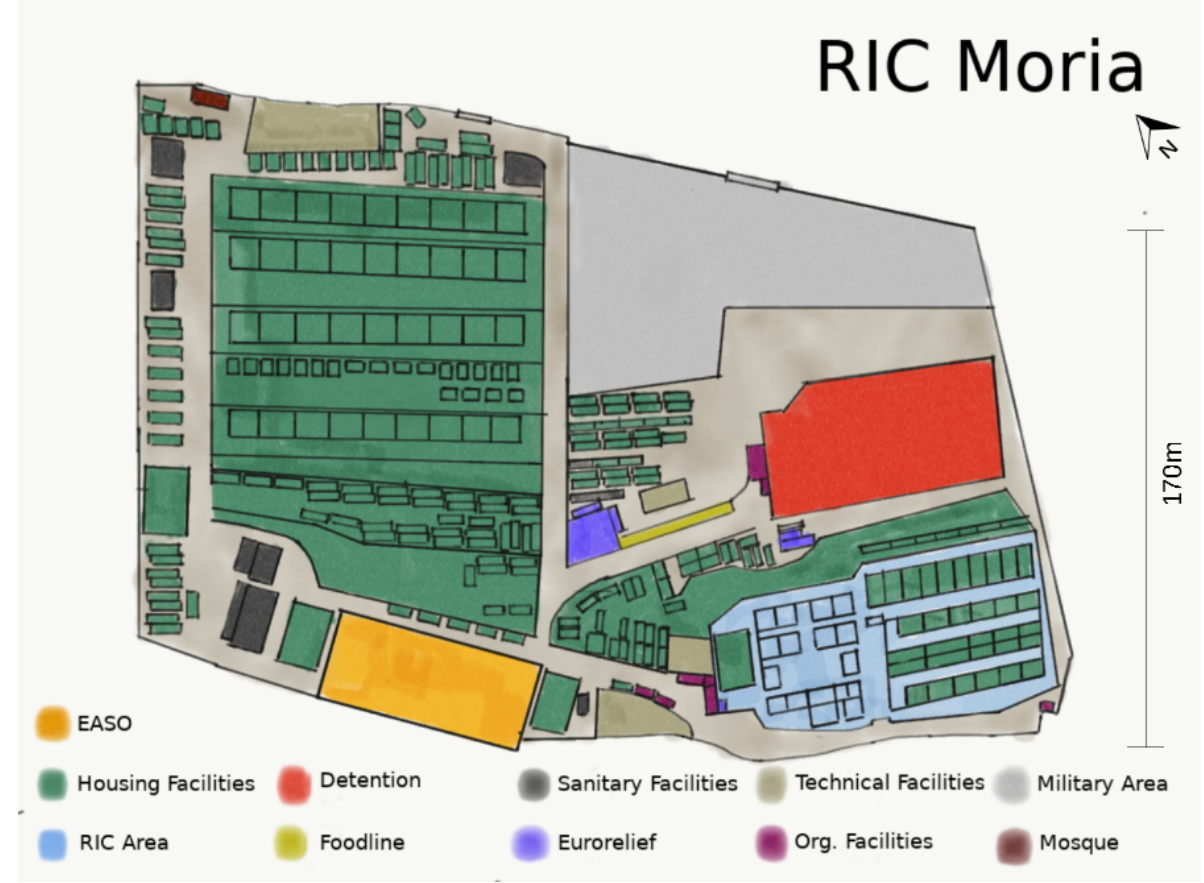

Abb. 1. Übersicht Reception and Identification Center Lesvos (eigene Abbildung).

stets über starke psychische Beschwerden geklagt hatte (Deportation Monitoring Aegean, 2020:o. S.).

Trotz der starken Einschränkungen bestand ein - wenn auch starker Regulierung unterworfener - Moment, in dem die Inhaftierten eigenständig mit dem Außen der Haftanstalt in Beziehung treten konnten: Zweimal täglich gab es anderthalb Stunden Hofgang. Die Zeit des Hofgangs variierte jedoch, sodass der Kontakt zu Mithäftlingen aus anderen Zellen im Außenbereich möglichst vermieden und Informationsfluss unterbunden wurde (Interview mit Amissah). Zusätzlich bestand während dieser Zeit die Möglichkeit, zu Außenstehenden durch die Absperrungen hindurch Kontakt aufzunehmen.

Die Immobilisierung wirkte sich zudem auf die Zeit nach der Haft aus: Während Einzelne dem Anschein nach schon vor der Haft traumatisiert waren, berichteten andere von Angstzuständen und Flashbacks, wenn sie in die Nähe der Hafteinrichtung kamen, die unmittelbar neben der Essensausgabe des Lagers lag. Die ehemals Inhaftierten klagten über Probleme, ihren Alltag unter den ohnehin schon schwierigen Umständen zu bestreiten, und erzählten von anderen ehemaligen Insassen, die starke posttraumatische Stresssymptome aufwiesen (Interview mit Ato).

\subsection{Zirkulation von Informationen und Gütern}

Die eingeschränkte Zirkulation von Informationen und Gütern bestimmte den Haftalltag der Interviewten. Alle mussten sich ohne Vorinformation in ihrer ursprünglichen Gruppe sammeln und wurden vom New-Arrival-Areal mit einem Ge- fängnisbus in die naheliegende Haftanstalt überstellt. Während nur einzelne Personen einer Leibesvisitation unterzogen wurden, mussten alle Inhaftierten ihre persönlichen $\mathrm{Ge}-$ genstände aushändigen, die dann inventarisiert wurden. Nach ihrer Durchsuchung wurden die neu Inhaftierten aufgefordert, ein auf Griechisch verfasstes Dokument zu unterzeichnen. Das Dokument wurde jedoch weder übersetzt noch wurde ihnen erklärt, was es beinhaltet (Interview mit Ato). Im Nachhinein stellte sich heraus, dass sie mit ihrer Unterschrift die Zustimmung zu ihrer eigenen Inhaftierung für einen Zeitraum von 90 Tagen gegeben hätten (Interview mit Yoofi). Demgegenüber hätten sie laut Saranti (2019) lediglich einer Inhaftierung von drei Tagen - zur Durchführung des Verwaltungsakts - zugestimmt. Auf die Frage, was passiert wäre, wenn sie das Dokument nicht unterzeichnet hätten, sagte Ato aus, dass sich ihnen diese Frage gar nicht gestellt habe. Kwesi sagte andererseits aus, dass die 90 Tage erst nach der Unterschrift gezählt worden seien. Zudem wurde von Aussagen anderer Gefangener berichtet, wonach Beamt*innen die Papiere nach ihrer Weigerung selbst unterschrieben hätten. Meist hätten die Inhaftierten erst von Mithäftlingen die Dauer und Umstände der Haft erfahren (Interview mit Amissah). Diese Tatsache kann als sehr prägnantes Beispiel dafür dienen, wie essenziell Informationen für Asylsuchende sind, um ihre Rechte einfordern, ihre Lage verbessern bzw. informiert handeln zu können.

Zudem sei der Zugang zu Versorgungs- oder Alltagsgütern erheblich eingeschränkt und von Willkür gekennzeichnet gewesen. So wurde bspw. berichtet, dass einzelne Zellen nur 
zwei Flaschen Wasser pro Tag für alle Insassen bekommen hätten. Bei einem anderen Beamten habe man für jedes Glas Wasser fragen müssen, während ein weiterer unbegrenzten Zugang zu Trinkwasser gewährt habe. Auch der Zugang zu Hygieneartikeln sei stark eingeschränkt gewesen, teils habe alles nachgefragt werden müssen. Es habe zwar regelmäßig Essen gegeben, jedoch sei die Ausgabe teils verzögert oder das Essen zu lange in der Sonne stehengelassen worden (Interview mit Ato). Selbst der Zugang zu persönlichen Medikamenten sei reguliert gewesen und hätte stets erfragt werden müssen, was Amissah als eine starke Einschränkung seines Autonomieempfindens wertete. Des Weiteren hatten die Inhaftierten keinen Anspruch auf die finanzielle Versorgungsleistung von zum Zeitpunkt der Interviews 90 Euro, sodass, selbst bei geringem Kontakt nach außen, beispielsweise keine SIM-Karten gekauft werden konnten.

Die Regulierung des Zugangs zu einem bestimmten persönlichen Gegenstand hatte für die Befragten eine enorme Bedeutung, da sie weitreichende Folgen für das Leben in Haft wie für das Asylverfahren und den Kontakt nach auBen hatte: Nur jeweils von Samstagmorgen bis Sonntagabend erhielten die Inhaftierten ihr Mobiltelefon. Die Beschränkung wirkte sich zum einen auf das Zeiterleben der Inhaftierten aus, deren Wochenende nach eigener Aussage durch den Kontakt zur Außenwelt deutlich erträglicher wurde (Interview mit Kwesi). Zum anderen fungierte das Mobiltelefon als Mittel, um Netzwerke aufrechtzuerhalten oder Beratungen zu organisieren, was erhebliche Auswirkungen auf die Chancen eines erfolgreichen Asylverfahrens hatte (s. nächster Abschnitt).

Die Zeit nach der Haft war für die meisten Inhaftierten eine Phase der Orientierungslosigkeit und des verminderten Wissens über örtliche (Unterstützungs-)Strukturen. So mussten einzelne Interviewte in der ersten Zeit nach der Haft auf der Straße in Moria schlafen, da sie weder von der mit der Unterbringung betrauten NGO noch über soziale Netzwerke untergebracht wurden. Die Interviewten wussten teilweise nicht, bei welchen Problemen sie sich an welche Organisation oder Institution zu wenden hatten, wo sich die Asylbehörden innerhalb des Lagers befanden oder welche Unterstützungsstrukturen außerhalb des Lagers existierten, sodass sich die Einschränkung der Handlungsmacht der Interviewten über die Haft hinaus eingeschrieben hat.

\subsection{Zugänge zu Institutionen und Unterstützungsleistungen}

Doch bereits während des konkreten Einschlusses hatten die Inhaftierten keinen Zugang zu essenziellen Institutionen und Unterstützungsleistungen - Gründe dafür waren die stark eingeschränkte Bewegungsfreiheit, die Abschottung nach außen und damit der fehlende Zugang zu Unterstützer*innen. Wie in der Chronologie kurz angerissen, begann dies bereits damit, dass teilweise keine medizinische und psychologische Voruntersuchung stattfand. Vorgesehen war, dass die Regis- trierung inklusive der Untersuchungen und der Ankündigung des Asylbegehrens noch vor der Haft durchgeführt wird, um festzustellen, ob die Personen von der Haft ausgenommen werden können. Dies hätte entweder aufgrund nachvollziehbarer Asylgründe der Fall sein können, vor allem aber aufgrund ihres Vulnerabilitätsstatus, ${ }^{3}$, der eine Überführung in das reguläre Verfahren ein- und damit eine Rücküberweisung in die Türkei ausschloss (Interview mit Anwältin 1).

Mehrere Autor*innen identifizieren fehlende Untersuchungen und Feststellungen der Vulnerabilität ebenfalls als substanzielles Problem der Wahrung der Rechte von potenziell Inhaftierten. So gibt Hänsel (2019) an, dass die Feststellung der Vulnerabilität aufgrund von Verfahrens- oder strukturellen Mängeln weder vor noch nach der Haft durchgeführt wurde und damit eine andauernde Inhaftierung nach sich zog (Hänsel, 2019:90), zumal andere Möglichkeiten der Feststellung außerhalb der Erstregistrierung und des Asylinterviews während der Inhaftierung nicht vorgesehen waren (Saranti, 2019:11). Dazu kam eine ebenfalls unzureichende medizinische und psychologische Versorgung, die unter anderem aus dem Einschluss der Inhaftierten resultierte. Es lässt sich zwar konstatieren, dass die unzureichende Gesundheitsversorgung auf Lesvos generell einen Einflussfaktor darstellte und mit dazu führte, dass Geflüchteten keine angemessene Behandlung zuteil wurde (und immer noch wird). Allerdings war die ärztliche Versorgung der Inhaftierten auch von der Willkür der Beamt*innen abhängig. Einige Personen wurden etwa in das nahegelegene Krankenhaus gebracht, um behandelt zu werden, während andere im gesamten dreimonatigen Haftzeitraum überhaupt keine Behandlung erhielten. Kwesi vermutete, dass die Polizei eine Haftentlassung durch Krankheit habe vermeiden wollen. Die psychologische Versorgung wurde als ähnlich desaströs beschrieben. Es sei zwar regelmäßig psychologisches bzw. sozialarbeiterisches Personal in die Hafteinrichtung gekommen, jedoch beschrieben die ehemaligen Inhaftierten dieses als für ihre Situation wenig hilfreich. Alle Befragten klagten zudem darüber, sich von den Beamt*innen nicht ernst genommen gefühlt zu haben, selbst wenn sie schon vor der Haft gesundheitliche Probleme angegeben hatten. Teilweise seien ihre Beschwerden ins Lächerliche gezogen worden (Interview mit Amissah).

Eine weitere, sich sowohl auf die Haftzeit selbst als auch auf das Asylverfahren auswirkende Beschränkung lag im fehlenden Zugang zu Anwält*innen. So war es den Inhaftierten kaum möglich, ohne Unterstützungsnetzwerke von außen anwaltliche Hilfe zu organisieren und sich etwa adäquat

\footnotetext{
${ }^{3}$,As vulnerable groups shall be considered for the purposes of this law: a) Unaccompanied minors, b) Persons who have a disability or suffering from an incurable or serious illness, c) The elderly, d) Women in pregnancy or having recently given birth, e) Single parents with minor children, f) Victims of torture, rape or other serious forms of psychological, physical or sexual violence or exploitation, persons with a post-traumatic disorder, in particularly survivors and relatives of victims of ship-wrecks, g) Victims of trafficking in human beings." (Asylgesetz 4375, Absatz 14, § 8)
} 
auf das Asylinterview vorzubereiten, das meist während der Haft stattfand (s. Abschnitt zu Mobilisierung). Zwar hätte eine Möglichkeit darin bestanden, selbstständig über das Mobiltelefon nach Anwält*innen zu suchen, jedoch durften die Inhaftierten ihr Mobiltelefon nur am Wochenende nutzen und waren unter der Woche nicht erreichbar. Einzelne Personen hatten nach Aussage von Ato bspw. nicht die Möglichkeit, ihren Anwalt bzw. ihre Anwältin zu sprechen, während sie kurzfristig einen Interviewtermin oder Ähnliches bekommen hätten. Auch konnten auf dem Handy befindliche Beweise für den Asylgrund während des Interviews nicht erbracht werden (Interview mit Yoofi). So wird deutlich, dass sich die unterschiedlichen Techniken der Regierung wie im vorliegenden Fall ergänzten und miteinander verschränkten, sodass die unterbundene Zirkulation von Informationen und Gütern auch zu einer Einschränkung des Zugangs zu Unterstützungsleistungen führte, die diesen Mangel möglicherweise hätten ausgleichen können.

Trotz alledem bestanden vereinzelt Möglichkeiten, Kontakte nach außen und zu Unterstützungsnetzwerken aufzubauen, die im Anschluss Anwält*innen oder bspw. SIM-Karten organisierten. Diese gingen jedoch meist auf schon bestehende Netzwerke zurück, etwa wenn Unterstützer*innen vorher von der Inhaftierung erfuhren oder gemeinsam mit den Inhaftierten auf der Insel angekommen waren.

\subsection{Mobilisierung und Beschleunigung der Zirkulation}

Zudem stützte sich die Regierung der Asylsuchenden nicht lediglich auf die Immobilisierung und die Unterbindung von Zugängen, sondern auch auf die bewusste und kontrollierte Mobilisierung sowie eine vor allem in Bezug auf das Asylverfahren beschleunigte Zirkulation, die sich mit anderen Techniken verschränkte.

So wurden Asylsuchende von Vollzugsbeamt*innen mobilisiert - meist zur Durchführung eines Asylinterviews bzw. zu Krankenhausbesuchen. Dabei wurden die Inhaftierten außerhalb der Haft stets in Handschellen transportiert, selbst bei Krankenhausbesuchen und Behandlungen. Die Beamt*innen hätten auf Beschwerden der Inhaftierten hin geäußert, so seien die Gesetze, sie müssten in Handschellen transportiert werden (Interview mit Amissah).

Während ein Großteil der Asylsuchenden im RIC Lesvos bis zu zwei Jahre auf das erste Asylinterview warten musste, gaben sich die Behörden bei Inhaftierten große Mühe, das Interview während der Haftzeit durchzuführen. Damit gingen einige Besonderheiten einher, hinter denen sich System vermuten lässt. So sorgte der beschränkte Zugang zu Anwält*innen während der Haft dafür, dass sich die Inhaftierten unzureichend auf das Interview (das ihnen den Asylstatus verschaffen sollte) vorbereitet fühlten. Neben den allgemeinen Haftbedingungen löste dies erhöhten Stress aus. So wurde in den Interviews gesagt, dass Inhaftierte nicht wussten, welche die richtige Formulierung zur Beantwortung der jeweiligen Fragen gewesen sei und was ihnen zu einem gesi- cherten Schutzstatus verholfen hätte (Interview mit Ato). Zusätzlich sorgte die weitestgehende Beschränkung von Kontakten dafür, dass die Inhaftierten keine Möglichkeiten hatten, Beweise für das Interview zu sammeln und vorzubereiten (Interview mit Amissah). Neben anwaltlichen Versuchen, das Interview auf nach der Haft zu verschieben, führte lediglich das Auftreten bzw. das Anhalten einer Krankheit zur Verschiebung des Interviewtermins, im besten Fall bis über die Haftzeit hinaus (Interview mit Ato). Die Möglichkeiten, den Rahmen für das Interview annehmbarer zu gestalten, waren für die Inhaftierten folglich stark eingeschränkt. Dies hatte in mehreren dokumentierten Fällen zur Folge, dass die ehemals Inhaftierten kurz nach Haftentlassung eine erstinstanzliche Ablehnung ihres Asylantrags erhielten. Im Anschluss daran blieb ihnen der Einspruch und ein zweites Verfahren oder die Deportation. Die Beschleunigung des Verfahrens, gepaart mit unzureichendem Zugang zu Informationen und Unterstützung, führte dementsprechend zu einer beschleunigten Zirkulation im übergeordneten Sinne, die Personen mit niedriger Anerkennungsquote schnellstmöglich aus dem Asylsystem herausbefördern sollte.

\subsection{Umgangsstrategien und Widerstand}

Auch wenn - so wie im vorliegenden Artikel - die Subjektivität und Handlungsmacht der Asylsuchenden stets betont werden, bestand in diesem Fall - etwa hinsichtlich der Verschiebung des Asylverfahrens - nur wenig Handlungsspielraum. Darauf würden sich laut Yoofi allerdings die meisten Anwält*innen konzentrieren, um mehr Zeit für die Vorbereitung des Asylverfahrens zu erwirken. Dass auftretende Krankheiten das Interview verzögern können, lässt sich in diesem Kontext wohl weniger als dezidierte Handlungsstrategie fassen, die Bemühung darum, dies als legitimen Grund anerkennen zu lassen, hingegen schon.

Wie bereits erwähnt, beschreibt der Hofgang einen der wenigen Momente, mit Personen und sozialen Netzwerken außerhalb in Beziehung zu treten, indem Bekannte an den Zaun des Außenbereichs treten und mit den Inhaftierten reden (Interview mit Ato). Teils versuchten die Beamt*innen, dies zu unterbinden, dann verständigten sich die Parteien jedoch über Zurufe. Informelle Netzwerke nach außen bildeten so eine zwar eingeschränkte, aber doch hilfreiche Ressource für die Inhaftierten. Während Besucher*innen sie nicht mit Lebensmitteln oder Ähnlichem versorgen durften, hatten Außenstehende die Möglichkeit, emotionale und finanzielle Unterstützung, bspw. für Medikamente oder SIM-Karten, zu leisten (Interview mit Kofi). Ein besonders großer Vorteil dieser Praxis bestand darin, dass der Zugang zu anwaltlicher Beratung von außen deutlich erleichtert wurde und die Unterbringung nach der Haft selbst organisiert werden konnte. 


\section{Fazit}

Bei rückblickender Betrachtung der Empirie lässt sich nun unter Rückgriff auf die Gouvernementalitätstheorie die Herausbildung bestimmter Systeme der Zirkulation auf kleinräumiger wie übergeordneter Ebene ausmachen, die durch die Verschränkung nicht nur - wie von Foucault identifizierter - liberaler Techniken der Regierung gewährleistet werden. Vor allem Techniken, die auf der Praxis des Einschlusses im Detention Center fußten, machten den beschriebenen Mechanismus aus. Dabei wurde zum einen die Verlangsamung oder die starke Einschränkung von Zirkulationen und zum anderen die Beschleunigung der Zirkulation vor allem der Inhaftierten angestrebt.

$\mathrm{Zu}$ diesem Zweck wurden die unterschiedlichen, in der Empirie genannten Techniken in einer Art und Weise relationiert, dass sie der Verminderung der Chancen auf Asyl für Personen mit niedriger Anerkennungsquote und damit einem spezifischen Zirkulationssystem für unerwünschte Asylsuchende dienten. Die vereinzelten Techniken lassen sich dabei nicht isoliert betrachten, sondern entfalteten ihre Wirkung hin zum Ziel der Regierung erst in ihrer Beziehung zueinander, aber auch im Zusammenspiel mit anderen Orten des Asylsystems. So führte die übergeordnete Technik des selektiven Einschlusses dazu, dass die Inhaftierten einen begrenzten und meist willkürlichen Zugang zu Informationen und Gütern besaßen, der sich mit dem geringen Zugang zu Unterstützungsleistungen und Netzwerken ergänzte und in ihrer Kombination eine prekäre Situation für die Inhaftierten hinsichtlich ihrer Asylverfahren schuf. Netzwerkbildungen, die den Asylsuchenden hätten dienen können, wurden also durch die Inhaftierung unterbunden. Gleichzeitig wurden durch eine gezielte und unter sehr strikten Umständen stattfindende Mobilisierung die Netzwerke, die dem Erreichen des Regierungsziels folgten, produziert. Dies beginnt mit dem Low Profile Detention Scheme, das eine bestimmte Gruppe von Asylsuchenden in das System der Detention einbindet und sich über die beschleunigten bzw. erschwerten Verfahren und damit niedrig bleibenden Anerkennungsquoten reproduzierte. Die gezielte und dieser beschleunigten Zirkulation dienende Mobilisierung drückte sich räumlich vor allem im Transport zu den Interviews unter den aus dem Einschluss resultierenden Einschränkungen und zeitlich in der zeitnahen Durchführung der Interviews aus und mündete auf übergeordneter Ebene bei ausbleibendem Erfolg seitens der Asylsuchenden in ihrer Deportation. Diese wird weiterhin entweder durch das angesprochene Abkommen mit der Türkei oder durch Rücknahmeabkommen mit den Herkunftsstaaten gewährleistet, sodass das Detention Center in ein größeres Netz der Prozessierung und Rückführung einer bestimmten Gruppe eingebunden war.

Diese Perspektive kann zum Anlass genommen werden, um über den zweiten Aspekt der Foucault'schen Theorie nachzudenken: der Verknüpfung und Relationierung lokaler Techniken und ihrer Eingebundenheit in größere Wirkungs- zusammenhänge. Dabei lassen sich unterschiedliche Ebenen räumlicher Reichweite ausmachen, deren Funktionieren nicht ohne ihre jeweilige Verschränkung bewerkstelligt werden könnte. Während konkret die Körper der Inhaftierten von unterstützenden Netzwerken isoliert und über erzwungene Mobilisierung in die beschleunigte Zirkulation eingebunden wurden, waren die Inhaftierten durch ihre mögliche Abschiebung in weitreichendere Netzwerke eingebunden, die durch ihre Inhaftierung und damit Lokalisierung nicht hätten aufrechterhalten werden können. Diese waren wiederum Teil einer weitreichenderen Rationalität und Migrationspolitik der EU, die auf übergeordneter Ebene die generelle Immobilisierung schon vor Eintritt in die EU anstrebte (s. z. B. EUTürkei-Deal). Falls die Asylsuchenden trotzdem die Möglichkeit der Einreise in die EU wahrnahmen, sollte über die in Beziehung stehenden Institutionen eine selektive Zirkulation erreicht werden, innerhalb derer vor allem als potenziell gefährlich Markierte oder aus vermeintlich wirtschaftlichen Gründen Migrierte aus Systemen der Zirkulation innerhalb Europas herausgefiltert werden sollten.

Was ist nun mit der im Artikel dargelegten Analysemethode gewonnen? Der konkrete Gewinn der Analyse liegt in der Aufdeckung der Relationierung konkreter Techniken, die zwar auch isoliert betrachtet werden können, ihre Wirkmächtigkeit aber erst in der Beziehung zu anderen Techniken entfalten. Ebenjene Wirkmächtigkeit konnte in der Analyse verdeutlicht werden. Was jedoch ebenfalls deutlich wurde, ist, dass sich die Technologien der Zirkulation bei der Unterbindung ebenjener nicht lediglich auf die liberalen Sicherheitsmechanismen des Anreizes und der Beeinflussung von Handlungsgrundlagen stützen. Vielmehr wird verlangsamte und beschleunigte Zirkulation innerhalb von Geflüchtetenlagern und vor allem in Detention Centers durch ein spezifisches Verhältnis zwischen kontrollierter Mobilisierung und Immobilisierung sichergestellt. Dies wiederum wird nur durch einen konkreten Zugriff auf den individuellen Körper der Asylsuchenden ermöglicht - Techniken, die eher an Disziplinarinstitutionen erinnern.

Abschließend kann festgehalten werden, dass sich die Zirkulationssysteme unterschiedlich ausdifferenzieren und sich nicht nur im klassischen Sinne verstandener liberaler Techniken bedienen, sondern - je nachdem, welchem Ziel die Zirkulationssysteme dienen sollen - auch Merkmale des Einschlusses, der Immobilisierung und der erzwungenen bzw. kontrollierten Mobilisierung aufweisen können. Es ließe sich in diesem Kontext also eher von mehreren Zirkulationssystemen reden, die durch unterschiedliche Techniken liberal, disziplinarisch, autoritär etc. produziert werden und ineinandergreifen, sodass selektive Zirkulationen geschaffen werden, die im Falle des Asylregimes bestimmte Personengruppen unter gegebenen Kategorien (z. B. hohe Verdunklungsgefahr, Wirtschaftsmigrant*in, vulnerabel) unterschiedlich einbindet und ihre Bewegung in unterschiedliche Richtungen (z. B. nach Athen/Europa oder durch die Rückführung wieder aus der EU heraus) beschleunigt, verlangsamt oder sogar tempo- 
rär stoppt. Das Detention Center als Raum des Einschlusses bildet im vorliegenden Fall einen zentralen Punkt eines dieser Zirkulationssysteme.

Danksagung. Ich möchte denen danken, die mich bei der Forschung vor Ort unterstützt und mir damit erst ermöglicht haben, jedoch vor allen den ehemaligen Inhaftierten, die trotz der schwierigen Lage Vertrauen in mich fassen konnten und ihre Geschichte mit mir geteilt haben.

Datenverfügbarkeit. Die Interviewdaten sind aus Gründen des Identitätsschutzes der Interviewpartner*innen nicht öffentlich einsehbar.

Interessenkonflikt. Die Autor*innen erklären, dass kein Interessenkonflikt besteht.

Haftungsausschluss. Copernicus Publications bleibt in Bezug auf gerichtliche Ansprüche in veröffentlichten Karten und institutionellen Zugehörigkeiten neutral.

Begutachtung. This paper was edited by Benedikt Korf and reviewed by two anonymous referees.

\section{Literatur}

Agamben, G.: Homo sacer - Die souveräne Macht und das nackte Leben, Suhrkamp, Frankfurt am Main, 2002.

Alford, C. F.: What would it matter if everything Foucault said about prison were wrong? Discipline and Punish after twenty years, Theor. Soc., 29, 125-146, 2000.

Altin, R. und Minca, C.: The ambivalent camp. Mobility and excess in quasi-carceral Italian asylum seekers hospitality centre, in: Carceral Mobilities, Interrogating Movement in Incarceration, Herausgeber*innen: Peters, K. und Turner, J., Routledge, New York, 30-43, 2017.

Bosworth, M.: Subjectivity and identity in detention: Punishment and society in a global age, Theor. Criminol., 16, 123-140, 2012.

Breuckmann, T.: Physische Barrieren als Elemente der Ordnung und Regierung von Geflüchteten. Ansätze einer geogouvernementalistischen Mobilitätsforschung am Beispiel Lesvos, in: Praktiken der (Im-)Mobilisierung. Lager, Sammelunterkünfte und Ankerzentren im Kontext von Asylregimen, Herausgeber*innen: Devlin, J., Evers, T., and Goebel, S., transcript, Bielefeld, 73-94, 2021.

Collier, S. J.: Topologies of Power: Foucault's Analysis of Political Government beyond "Governmentality", Theor. Cult. Soc., 26, 78-108, 2009.

Conlon, D. and Hiemstra, N.: Examining the everyday microeconomies of migrant detention in the United States, Geogr. Helv., 69, 335-344, https://doi.org/10.5194/gh-69-335-2014, 2014.
Deportation Monitoring Aegean: Cruel Detention Policies in Greece on the Rise - Death in Moria Prison shows the Violence of Migrant Incarceration System in the European Hotspots, available at: https://dm-aegean.bordermonitoring.eu/2020/01/18/crueldetention-policies-in-greece-on-the-rise-death-in-moria-prisonshows-the-violence-of-migrant-incarceration-system-in-theeuropean-hotspots/ (letzter Zugriff: 15 November 2021), 2020.

Fili, A.: Containment Practices of Immobility in Greece, in: Critical Perspectives on Migration in the Twenty-First Century, Herausgeber*innen: Karakoulaki, M., Southgate, L., and Steiner, J., E-International Relations, Bristol, 162-179, 2018.

Fishwick, E. and Wearing, M.: "Unruly mobilities" in the tracking of young offenders and criminality. Understanding diversionary programs as carceral space, in: Carceral Mobilities. Interrogating Movement in Incarceration, Herausgeber*innen: Peters, K. and Turner, J., Routledge, New York, 44-56, 2017.

Forman, P. J.: Circulations beyond nodes: (in)securities along the pipeline, Mobilities, 13, 231-245, 2018.

Foucault, M.: Überwachen und Strafen. Die Geburt des Gefängnisses, Suhrkamp, Frankfurt am Main, 1976.

Foucault, M.: Die Geburt der Biopolitik. Geschichte der Gouvernementalität II, Suhrkamp, Frankfurt am Main, 2015.

Foucault, M.: Überwachen und Strafen. Die Geburt des Gefängnisses, in: Foucault, M.: Die Hauptwerke, Suhrkamp, Frankfurt am Main, 701-1019, 2016.

Foucault, M.: Sicherheit, Territorium, Bevölkerung. Geschichte der Gouvernementalität I, Suhrkamp, Frankfurt am Main, 2017.

Füller, H. und Michel, B.: Einleitung: Raum als Heuristik für die sozialwissenschaftliche Machtanalyse, in: Die Ordnung der Räume. Geographische Forschung im Anschluss an Michel Foucault, Herausgeber*innen: Füller, H. und Michel, B., Westfälisches Dampfboot, Münster, 7-22, 2012.

Gordon, C. (Hg.): Michel Foucault, Power/Knowledge, Selected Interviews and Other Writings 1972-1977, Pantheon Books, New York, 1980.

Hänsel, V.: Gefangene des Deals. Die Erosion des europäischen Asylsystems auf der griechischen Hotspot-Insel Lesbos, o. O., online aufrufbar: https://bordermonitoring. eu/wp-content/uploads/2019/05/bm.eu-2019-aegean.pdf (letzter Zugriff: 16 November 2021) 2019.

Hsu, H.-F.: The Control Model in a Mega-Prison: Governing Prisons in Taiwan, International Criminal Justice Review, 13, 149167, 2003.

Huxley, M.: Space and Government: Governmentality and Geography, Geography Compass, 2, 1635-1658, 2008.

Inhetveen, K.: Die politische Ordnung des Flüchtlingslagers. Akteure - Macht - Organisation. Eine Ethnographie im Südlichen Afrika, transcript, Bielefeld, 2014.

mare liberum: Moria 2.0 - The reproduction of inhumanity, 25.09.2020, available at: https://mare-liberum.org/en/ news/moria-2-0-the-reproduction-of-inhumanity (letzter $\mathrm{Zu}$ griff: 15 November 2021), 2020.

Marquardt, N. und Schreiber, V.: Die Neue Kulturgeographie und Foucault. Arbeit mit und in gemischten Zuständen, in: Die Ordnung der Räume. Geographische Forschung im Anschluss an Michel Foucault, Herausgeber*innen: Füller, H. und Michel, B., Westfälisches Dampfboot, Münster, 23-53, 2012. 
Martin, D., Minca, C., und Katz, I.: Rethinking the camp: On spatial technologies of power and resistance, Prog. Hum. Geog., 2019, 1-26, 2019.

Mincke, C. und Lemonne, A.: Prison and (im)mobility. What about Foucault?, Mobilities, 9, 528-549, 2014.

Moran, D., Piacentini, L., und Pallot, J.: Disciplined mobility and carceral geography: prisoner transport in Russia, Transactions of the Institute of British Geographers, 37, 446-460, 2010.

Moran, D., Turner, J., und Schliehe, A.: Conceptualizing the carceral in carceral geography, Prog. Hum. Geog., 5, 666-686, 2018.

Mountz, A., Coddington, K., Catania, R. und Loyd, J.: Conceptualizing detention: Mobility, containment, bordering, and exclusion, Prog. Hum. Geog., 37, 522-541, 2012.

Mountz, A. and Loyd, J.: Transnational productions of remoteness: building onshore and offshore carceral regimes across borders, Geogr. Helv., 69, 389-398, https://doi.org/10.5194/gh-69389-2014, 2014.
Oesch, L.: The refugee camp as a space of multiple ambiguities and subjectivities, Political Geography, 60, 110-120, 2017.

Pasquetti, S.: Negotiating control. Camps, cities and political life, City, 19, 702-713, 2015.

Peters, K. und Turner, J.: Carceral mobilities. A manifesto for mobilities, an agenda for carceral studies, in: Carceral Mobilities. Interrogating Movement in Incarceration, Herausgeber*innen: Peters, K. und Turner, J., Routledge, New York, 1-13, 2017.

Philo, C.: Foucault's geography, Environ. Plann. D, 10, 137-161, 1992.

Salter, M.: To Make Move and Let Stop: Mobility and the Assemblage of Circulation, Mobilities, 8, 7-19, 2013.

Saranti, E.: Locked up without rights. Nationality-based detention in the Moria refugee camp, HIAS Policy Brief, December 2019, o. O., online aufrufbar: https://www.hias.org/sites/default/files/ report_on_low_profile_detention_in_greece_hias_dec_2019.pdf (letzter Zugriff: 16 November 2021), 2019. 